\title{
Inserção do fisioterapeuta no Programa Saúde da Família
}

\author{
The physiotherapist insertion in the family health program
}

Kéllin Daneluz Delai ${ }^{1}$

M iriam Salete W ilk W isniewski ${ }^{2}$

${ }^{1}$ UNIM ED Erechim. Rua Itália 969, Centro. 99700-000 Erechim RS. kellindd@hotmail.com ${ }^{2}$ Curso de Fisioterapia, Universidade Regional Integrada do Alto U ruguai e das M issões.
Abstract Study of quali-quantitative character that had as its main objective, to map out the Physiotherapist insertion in the Family Health Program (PSF) of the cities that compose a Regional Coordination of $\mathrm{H}$ ealth (CRS) in Rio Grande do Sul State. For this, after theidentification of the cities that compose the referred coordination and professional physiotherapists working in these cities, it was sent, via e-mail, a pre-prepared questionnaire, containing opened and closed questions about the subject. The study was done in the period between August and October 2007. The obtained results showed that in the cities under study, no physiotherapist is inserted into the PSF, there are cities that do not provide the physiotherapy service, and the perception of professional physiotherapists about their participation in the Program is directed to the interest in the interaction with the community, preventive way. The conclusion is that there is no knowledgeabout the professional physiotherapist skills from the municipal managers, lack of professional recognition in order of preventive work, and the physiotherapy as health field with so important participation as the others, inserted in PSF. Already, in the context of new existing $\mathrm{H}$ ealth Politics, the physiotherapy has important place to the performance in the community group.

Key words Family Health Program, Physiotherapy, Primary prevention
Resumo Estudo decaráter qualiquantitativo que teve como objetivo principal mapear a inserção do fisioterapeuta no Programa de Saúde da Família (PSF) dos municípios que compõem uma Coordenadoria Regional de Saúde (CRS) no Rio Grande do Sul. Para tanto, após a identificação dos municípios que compõem a referida coordenadoria e profissionais fisioterapeutas atuantes nessas cidades, enviou-se, via e-mail, um questionário préelaborado, contendo perguntas abertas e fechadas acerca da temática. 0 estudo foi realizado no período de agosto a outubro de 2007. O sresultados obti dos demonstraram que, nos municípios em estudo, nenhum fisioterapeuta está inserido no PSF. Existem municípios que não disponibilizam o serviço de fisioterapia. E a percepção dos profissionaisfisioterapeutas, acerca desua participação no programa está direcionada ao interesse na atuação junto à comunidade de forma preventiva. Conclui-se, assim, quehá um desconhecimento sobre as competências do profissional fisioterapeuta por parte dos gestores municipais e falta de reconhecimento do fisioterapeuta no que diz respeito à atuação preventiva e da fisioterapia como área da Saúde com participação tão importante quanto às demais, inseridas no PSF. Já no contexto das novas políticas de saúde vigentes, a fisioterapia tem lugar importante no que diz respeito à atuação na coletividade. Palavras-chave Programa Saúdeda Família, Fisioterapia, Prevenção primária 


\section{Introdução}

Há mais de duas décadas, o Brasil vem redefinindo o perfil do sistema de saúde. Nos anos noventa, reformularam-se os papéis e as funções dos entes governamentais na oferta de serviços, na gerência de unidades ena gestão do sistema de saúde.

Partilhar das decisões é um caminho para implementar o princípio ético da autonomia dos indivíduos e da coletividade. A conquista legal afirma o direito do cidadão à saúde e o dever do Estado está em garantir esse direito. 0 princípio da responsabilidade também cria, para o cidadão, uma obrigação de natureza ética de participar da tomada de decisões de natureza pública, para garantir a implementação de medidas que possam efetivar a humanização da aten ção à saúde, como propõe a U NICEF' " "prover bens e serviços, de modo diferenciado, para atender às distintas necessi dades dos vários grupos populacionais tendo em vista a redução das desigualdades de acesso".

Prevenção, na área de saúde, apesar dos problemas em conceituá-la, é certamente mais abrangente do que sua definição - ato de evitar que algo aconteça - e deve fazer parte da história natural da doença. Assim, distinguem-se três níveis de aplicação de medidas preventivas: atenção primária, secundária e terciária².

Para Baduy e Oliveira3, o Sistema Único de Saúde (SUS) tem apresentado resultados positivos nos propósitos de universalização, descentralização e ampliação da cobertura dos serviços de saúde. Avança, ainda com dificuldade, na garantia de qualidade, equidade ena resolutividade das assistências ambulatorial e hospitalar, principalmente pela falta de profissionais habilitados para prestar assistência integral à saúde. 0 alcance desses objetivos depende, dentre outras medidas, do incentivo à educação permanente como estratégia na reorganização dos serviços de saúde e da integração de todos os membros envolvidos no processo de construção de saúde para todos.

A estratégia do PSF propõe rever práticas, valores e conhecimentos de todos os envolvidos: amplia as ações a serem desempenhadas pelos profissionais de saúde e aumenta os limites e as possibilidades de atuação, exigindo novas habilidades e aptidões4.

A lógica que norteia o trabalho do PSF é a promoção da saúde, ou seja, os indivíduos e famílias devem ser assistidos antes do surgimento dos problemas e agravos de sua saúde. Seu trabalho deve priorizar a atenção básica, as ações de prevenção e a promoção da saúde e estabelecer uma relação permanente entre os profissionais de saúde e a população ${ }^{5}$.

Diante desse cenário, cabe à fisioterapia uma releitura de seus fundamentos e análise de suas práticas, com vistas a adaptar-se a essa realidade e contribuir na mudança do quadro social e sanitário do país. Em consonância com os princípios propostos pelo modelo de Vigilância à Saúde, o fisioterapeuta deve ser inserido em outros níveis deatenção e desenvolver suas ações deacordo com as diretrizes da territorialização eda adscrição da clientela6.

\section{M étodo}

Este estudo, de caráter qualiquantitativo, teve como foco os fisioterapeutas que atuavam em 31 municípios pertencentes a uma Coordenadoria Regional de Saúde, localizada na região norte do Rio Grande do Sul.

0 instrumento de pesquisa contou com um questionário que foi enviado a cada profissional fisioterapeuta via e-mail, correio ou entregueem mãos, contendo perguntas abertas e fechadas. Estas envolviam dados demográficos, de formação acadêmica e de atuação profissional. 0 período utilizado para a coleta dos dados envolveu os meses de agosto a outubro de 2007.

Foram enviados 39 questionários a profissionais de 31 municípios.

Para a análise quantitativa dos dados, utilizou-se a análise descritiva, enquanto que, para as questões com abordagem qualitativa, seguiuse o protocolo de análise de conteúdo.

Com relação aos aspectos éticos, o projeto foi submetido ao Comitê de Ética em Pesquisa e aprovado em 18 de julho de 2007.

\section{Resultados ediscussão}

Foram enviados 39 questionários a profissionais de 31 municípios que compõem a referida Coordenadoria Regional de Saúde. Destes, 24 retornaram, sendo a amostra desse estudo composta, então, por esse número de questionários. Do total de questionários enviados, quinze não retornaram, inviabilizando a análise do perfil sociodemográfico e formativo dos profissionais.

Identificou-seque, dentreos 31 municípios, oito não disponibilizavam o serviço de fisioterapia.

Quando o município não disponibiliza o serviço de fisioterapia, os indivíduos que necessi- 
tam desse tipo de tratamento precisam ser deslocados ao Centro de Referência, no caso em especial, à cidade-sede da CRS em questão. N o território estudado, existem municípios com localização muito distante do Centro de Referência, e outros, além da distância, não possuem acesso asfáltico. Levando-se em consideração as patologias tratadas, geralmente envolvendo dor e incapacidades físicas, e mais, o número de sessões solicitadas e necessárias para o pronto restabele cimento, esse deslocamento por muitas vezes é inviável e vai contra todo o princípio de tratamento fisioterapêutico proposto.

Segundo Harris et al. ${ }^{7}$, os municípios têm demonstrado dificuldades para garantir o acesso da população aos outros níveis de assistência, especialmenteno nível secundário. Os municípios de pequeno e médio portes têm conseguido ampliar, relativamente, a cobertura do Programa Saúde da Família (PSF); no entanto, encontram dificuldades no estabelecimento deum sistema regionalizado de referência e contrarreferência, que garanta uma assistência integral à população. Diante desse conceito, a aproximação entre a fisioterapia e o nível primário seria uma alternativa capaz de fortalecer a atenção básica, aumentando a resolutividade do sistema e contribuindo para a garantia da integralidade do mesmo.

De acordo com Unglert ${ }^{8}$, com a atuação dentro de um território estabelecido e com uma população definida, o fisioterapeuta passa a ter a possibilidade deacompanhar mais proximamente e ser responsável pela saúde da população adscrita. A lógica da responsabilização estimula o de senvolvimento de novas relações entre os profissionais e usuários, com o estabelecimento devínculos e a possibilidade de um acompanhamento continuado, o que potencializa o desenvolvimento de ações promocionais e preventivas.

Demograficamente, dos 24 fisioterapeutasque responderam ao questionário proposto, dezenove $(79,17 \%)$ são do sexo feminino e cinco, $(20,83 \%)$ do masculino. A idade variou de 23 a trinta anos, totalizando $83,34 \%$, isto é, a maioria dos profissionais; de trinta a quarenta anos, 8,33\%; e acima de quarenta anos, a mesma percentagem, ou seja, 8,33\% dos profissionais.

De acordo com o tempo de formação, obtiveram-se 21 profissionais ( $87,5 \%$ ) formados em um período entre dois e dez anos; etrês (12,5\%) formados há mais de dez anos. Dentreasuniversidades formadoras estão a U niversidade do O este de Santa Catarina (UNOESC/SC), Universidade do Vale do Itajaí (UNIVALI/SC), Universidade de Cruz Alta (UNICRUZ/RS), Universidade do Contestado - Concórdia (UnC/SC), Universidade de Passo Fundo (UPF/RS), Centro Universitário Feevale (FEEVALE/RS), UniversidadeFederal de Santa M aria (UFSM /RS) e Pontifícia Universidade Católica do Paraná (PUC/PR).

Ao questionar-se se, em sua formação, haviam tido noções sobre atenção primária, secundária eterciária à saúde, dezenove $(79,17 \%)$ responderam que sim e cinco, (20,83\%) não. Já para a pergunta que questionava se, na formação acadêmica, haviam recebido noções sobreo PSF, oito (33,33\% dos profissionais) informaram têlas recebido, sendo que, destes, quatro concluíram sua formação há menos de quatro anos; porém, a grande maioria dos profissionais questionados, isto é, dezesseis $(66,67 \%)$ responderam não haver recebido, em sua formação, noções acerca do Programa Saúde da Família.

Ao analisarmos a Tabela 1, referente à relação entrea faixa etária etempo deformação, fazse uma reflexão com relação ao tempo de implantação do Programa Saúde da Família no Brasil, que data do ano de 1994, ou seja, treze anos atrás. N este contexto, observou-se que, dos componentes da amostra, três profissionais apresentaram tempo de formação superior a este, 0 quetorna óbvio, a esses, o desconhecimento acerca do programa, em sua formação acadêmica.

No que se refere aos conhecimentos necessários às novas práticas profissionais, o fisioterapeuta deve se aproximar de saberes da epidemiologia, que oferece conhecimentos quanto à distribuição das doenças nas coletividades, fatores derisco; e das ciências sociais, quetrazem conhecimento dos fatores culturais, comportamentais e religiosos do processo saúde-doença. Com relação à interação com as outras áreas de saúde, 0 fisioterapeuta deve ter uma aproximação da vigilância sanitária, vigilância epidemiológica, vigi-

Tabela 1. Relação entre faixa etária e tempo de formação.

\begin{tabular}{lcrc}
\hline $\begin{array}{l}\text { Faixa } \\
\text { etária }\end{array}$ & Frequência & $\%$ & $\begin{array}{c}\text { Tempo de } \\
\text { formação - anos }\end{array}$ \\
\hline $20-25$ & 05 & 20,84 & $2 \pm 1$ \\
$25-30$ & 15 & 62,5 & $3 \pm 2$ \\
$31-35$ & 01 & 4,17 & 8 \\
$36-40$ & 01 & 4,17 & 15 \\
$41-45$ & 02 & 8,33 & $15 \pm 2$ \\
Total & 24 & 100 & - \\
\hline
\end{tabular}


lância nutricional, vigilância à saúde do trabaIhador e vigilância ambiental, com vistas à identificação e acompanhamento de problemas que requerem atenção contínua, ação sobre territórios definidos earticulação en tre ações promocionais, preventivas, curativas e reabilitadoras ${ }^{6}$.

Para Campos e Belisário ${ }^{9}$, entre os distintos problemas que afloram com a implantação do PSF, nenhum é mais grave do que a carência de profissionais em termos quantitativos e qualitativos para atender a essa nova necessidade. Contudo, todos esses elementos só fazem aprofundar o desafio ao se discutir o processo deformação e educação continuada desses profissionais, agora organizados em forma de equipe.

Ainda para os mesmos autores, em pauta, estão colocadas algumas modalidades de formação como o curso de Especialização em Saúde da Família, dirigido a profissionais de nível superior, e as propostas de capacitação da equipe em conjunto, proporcionando, dessa forma, uma formação voltada para as propostas das políticas vigentes e, mais especificamente, do PSF.

Com relação à carga horária semanal dedicada à profissão, observou-se que um $(4,17 \%)$ profissional dedica oito horas semanais à função de fisioterapeuta; oito profissionais (33,33\%) dedicam dezesseis horas semanais a sua profissão; nove profissionais (37,5\%), vinte horas semanais; três ( $12,5 \%)$, trinta horas semanais etrês profissionais (12,5\%), quarenta horas semanais, sendo que estes últimos executam sua atividade laboral na docência.

A carga horária semanal torna-se insuficiente para atender à demanda, ou seja, para um trabalho ser resolutivo e solucionar os problemas de saúde prevalentes na população, seria preciso tempo integral de dedicação, no qual poderiam ser desenvolvidas ações preventivas, no controle de riscos, e ações curativas, no controle de danos. Resume-se isso a um só termo: valorização da profissão, ou seja, desconhecimento, por parte da gestão municipal, das competências profissionais do fisioterapeuta. E, para contrabalançar isso, há necessidade de romper 0 isolamento eo individualismo da prática fisioterapêutica reabilitadora e atuar em equipe multiprofissional. 0 fisioterapeuta precisa atuar e interagir com profissionais daárea da saúdee deoutrasáreas, como sociólogos, antropólogos, estatísticos, educadores, engenheiros, buscando, assim, tornar-se conhecedor do universo em que atua e tornar conhecida sua profissão junto à sociedade.

De acordo com a forma de atuação profissional ou forma de prestação de serviços, obtive- ram-se dez fisioterapeutas $(41,67 \%)$ trabalhando em clínicas de fisioterapia estruturadas pelos próprios municípios; cinco (20,83\%) que prestam serviços terceirizados e, para isso, utilizam suas clínicas particulares; dois (8,33\%) que trabalham em clínica particular e não prestam serviço ao município; e sete $(29,16 \%)$ que trabaIham em outras atividades, dentre estas, o ensino superior. Ao se falar em prestação de serviços, tanto na clínica particular quanto na clínica estruturada pelo município, observa-se a contratação de forma terceirizada do serviço, pela qual o profissional écontratado por um número préestabelecido de horas e/ou salário préfixado pelo contrato e por um período de tempo que geralmentenão excede um ano, com possível renovação contratual, ou rescisão do mesmo, se assim o contratante avaliar. De acordo com os entrevistados, o contrato era geral mentedeprestação de serviços de fisioterapia e não incluía o Programa Saúde da Família. Desta forma, a atuação junto à equipe multiprofissional ocorria de maneira simultânea, quando estes trabalhavam na mesma Unidade Básica de Saúde e conseguiam interagir acerca das ações que cabem a cada um dos profissionais.

0 questionário continha, ainda, perguntas referentes à atenção primária, secundária eterciária. A questão versava sobre a atuação dos profissionais em cada uma das três linhas de atenção à saúde e, neste, obtiveram-se os seguintes resultados: para a atenção primária, quatorze fisioterapeutas $(58,33 \%)$ responderam que sim (atuavam) e dez (41,67\%) não atuavam na atenção primária; para a atenção secundária, tive mos $21(87,5 \%)$ profissionais atuantes e três (12,5\%) não atuantes; e, para a atenção terciária, $21(87,5 \%)$ que atuavam e três ( $12,5 \%)$ que não atuavam, por exercerem a docência.

Conforme a Figura 1, a atuação na atenção secundária e na terciária é relativamente maior em relação à atenção primária. Percebeu-se, dessa maneira, que uma pequena parte da amostra trabalha na prevenção das patologias, com atuação direta na comunidade.

Para Bispo Júnior ${ }^{10}$, enquanto a fisioterapia reabilitadora concentra sua atuação quase que exclusivamente no controle de danos, seja buscando a cura de determinadas doenças, reabilitando indivíduos com limitações ou desenvolvendo a capacidade residual funcional de indivíduos, com lesões irreparáveis, a fisioterapia coletiva possibilita e incentiva a atuação no controle de riscos, ou seja, de fatores que podem contribuir potencialmente no desenvolvimento da do- 


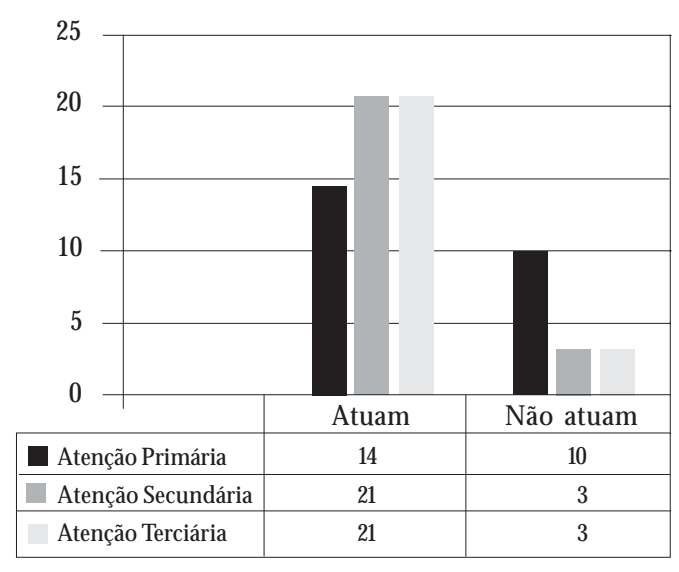

Figura 1. Atuação profissional nos três níveis de atenção à saúde.

ença. A diferença crucial éque, enquanto no controle de danos, a intervenção ocorre quando a doença já está instalada, muitas vezes em estado avançado, no controle de riscos a intervenção ocorre antes da doença acontecer, ou seja, antes do aparecimento de sequelas e limitação dos movimentos.

Faz-se necessário promover um profundo processo de mudança, que enlaceos estudantes à condição de sujeitos do processo de aprendizagem, que tome como ambientes de prática a comunidade, o domicílio e a Rede Básica de Saúde. Os profissionais ainda são preparados dentro de uma concepção que privilegia as abordagens individuais, curativas e centradas no hospital, clínica, consultório. São, portanto, incapazes de transcender o espaço do consultório e propor diagnóstico e intervenções sobre o coletivo, em uma abordagem integralizadora, sem a qual dificilmente se lograrão avanços significativos ${ }^{9}$.

Cada profissional da Equipe de Saúde da Família é fundamental para criar vínculos com a comunidade e ter funções específicas; porém, é importante o trabalho em equipe multiprofissional, a fim de assegurar a qualidade, eficiência e resolutividade que se esperam no PSF ${ }^{11}$.

Dentro desse contexto, o M inistério da Saú$\mathrm{de}^{4}$ destaca que, para obter melhor impacto sobre os diferentes fatores que interferem no processo saúde-doença, é importante que as ações tenham por base uma equipe formada por profissionais de diferentes áreas, capazes de atender diferentes demandas. A ação entrediferentes profissionais pressupõe a possibilidade da prática de um se reconstruir na prática do outro. Para lidar com a dinâmica da vida social das famílias assistidas e da própria comunidade, além de procedimentos tecnológicos específicos da área de saúde, a val orização dos diversos saberes e práticas contribui para uma abordagem mais integral e resolutiva.

No que dizia respeito à atuação na equipe de PSF, observou-se que 24 profissionais, ou seja, $100 \%$ da amostra, não atuavam nas equipes de PSF; porém dois, dentre estes, disseram atuar como equipe de apoio e um informou atuar somente em ações isoladas, como palestras sobre educação postural para escolares, atendimento domiciliar a pacientes acamados, participação em grupos de gestantes e diabéticos, com aulas de educação em saúde.

Para Machado ${ }^{12}$, no desenho brasileiro de equipe mínima, conforme o significado da palavra "mínima", há sugestões que autorizam vários outros tipos de equipe, que poderiam ser de oito a dez profissionais de saúde, mais os agentes comunitários, desde que cumpram as diretrizes e as tarefas propostas pelo SUS. Existem, ainda, equipes de apoio, como a de reabilitação, saúde mental e de promoção social.

A autora acredita ainda que, antes de se pensar em agregar qualquer outro profissional, deve-seter em mente a prioridade, queéa equipe mínima, já que a atenção básica é direito universal. Depois, haveria a complementação dos apoios matriciais. Porém, reconhece a necessidade de outros profissionais e, por isso, propõe ao $\mathrm{Mi}$ nistério da Saúde que retome a idéia de implantação dos Núcleos de Atenção Integral, aprovadana Comissão Intergestores Tripartide. Ela propõe aos municípios a contratação de equipes multiprofissionais em apoio às equipes de Saúde da Família. Dessa forma, os profissionais contemplados nos núcleos poderiam dar apoio e suporte técnico às equipes de PSF.

E por último, ao serem questionados sobresua opinião acerca da inserção do fisioterapeuta na equipe mínima de PSF, os profissionais demonstraram interesse na atuação como atenção primária à saúde: "O fisioterapeuta vem somar na equipe pela atuação junto à comunidade, principalmente na Atenção Primária. A inserção depende de pactuações, mas enfim, o profissional deve trabalhar sim preventivamente dentro da necessidade do munićpio em que atua" . (Fisioterapeuta 6)

"M uito importante, tanto quanto os outros profissionais, prevenindo muitas enfermidadesque podem ser evitadas com orientações adequadas". (Fisioterapeuta 2) 
Dentre as atribuições dos profissionais das equipes de Saúde da Família, destacam-se: prestar assistência integral à população, coordenar, participar e/ou organizar grupos de educação para a saúde, realizar visitas domici liares de acordo com o planejamento, fomentar a participação popular, discutindo com a comunidade conceitos de cidadania e direito à saúde e as suas bases legais ${ }^{3}$.

A fisioterapia, embora, ao longo da história, tenha se mantido no nível da reabilitação, possui competências ehabilidades suficientes para a atuação em outros níveis. Nesse sentido, apresentam-se algumas possibilidades de atuação do fisioterapeuta na atenção básica e em ambiente comunitário, deacordo com as diretrizes do PSF.

Para Bispo Junior ${ }^{10}$, é possível o fisioterapeuta desenvolver açõesjunto ao PSF utilizando seus saberes tão vastos na área da Saúde:

- Vigilância dos distúrbios cinesiofuncionais: constitui-seem subárea da Vigilância Epidemiológica e é responsável pelo acompanhamento e monitoramento da integridade físico-funcional e dos distúrbios relacionados à locomoção humana. Dessa forma, o objetivo é fornecer dados einformações relevantes às equipes de saúde, tanto na definição do perfil epidemiológico da população, como subsidiando as atividades de planejamento eintervenções. Deacordo com o atual cenário epidemiológico, al gumas condições apresentam maior potencial para a limitação da capacidade funcional das populações, a exemplo das patologias relacionadas ao aparelho cardiocirculatório, às causas externas e ao aumento da população idosa, às doenças cerebrovasculares, que deixam sequelas que levam à limitação da locomoção humana ou incapacidade física e os acidentes detráfego que, além de produzirem um número considerável deóbitos, têm gerado grandes contingentes de indivíduos com limitações irreversíveis e permanentes;

- Orientações posturais: a postura deve ser trabalhada como uma atitude corporal inerentea uma visão saudável efator preventivo para diversas doenças. $\mathrm{Na}$ atenção básica, o fisioterapeuta deve atuar com grupos populacionais, orientando sobre as posturas mais adequadas para cada grupo ou para cada situação. Deve-se levar em consideração quais os hábitos, costumes e crenças com poder de influência na postura daquela comunidade. Dessa forma, o fisioterapeuta deve atuar na orientação postural de escolares de forma continuada, destacando a participação dos professores. No período de gestação, ocorrem importantes repercussões corporais na marcha e no retorno venoso, dores lombares e desconforto respiratório. Em virtude dessas alterações, 0 fisioterapeuta deve atuar em grupos de gestantes, orientando quanto às posturas corporais, exercícios de alongamento, relaxamento e auxílio ao retorno venoso, orientações sobre exercícios respiratórios, além de incentivo ao aleitamento materno e orientações dos cuidados do bebê.

No que se refere à população idosa, a atuação do fisioterapeuta, na atenção básica, possibilita o desenvolvimento de ações relacionadas à melhora da postura, o estado físico-funcional, além de estimular o bem-estar. 0 fisioterapeuta deverealizar orientação da postura corporal; exercícios de relaxamento, alongamento e auxílio ao retorno venoso; caminhadas e atividades físicas moderadas; orientação quanto ao posicionamento adequado do mobiliário no lar, banheiros e dispositivos auxiliares, dentre outros;

- Desenvolvimento da participação comunitária: o fisioterapeuta, juntamente com os demais membros da equipe, deve estimular a criação dos Conselhos Municipais de Saúdetendo como referência aUnidade Básica deSaúde, com população e território adscritos. Dessa forma, o fisioterapeuta deve atuar no âmbito comunitário, incentivando e estimulando a participação da comunidade nas questões relacionadas à saúde;

- Desenvolvimento de ambientes saudáveis e incentivos a estilos de vida saudáveis: a atuação do fisioterapeuta, no desenvolvimento de ambientes saudáveis, perpassa por ações desenvolvidas junto aos indivíduos, às famílias eà comunidade, objetivando promover condições dignas de vida e saúde. 0 fisioterapeuta atuará, principalmente, no desenvolvimento de hábitos de vida saudáveis, tais como incentivo à prática da atividade física regular; adoção de hábitos alimentares saudáveis; combate ao tabaco, ao álcool e às drogas ilícitas; desestímulo à promiscuidade e estabelecimento de relações parentais estáveis; educação sexual para jovens e adultos, e incentivo à valorização e corresponsabilização da própria saúde da comunidade. Dessa forma, o fisioterapeuta contribuiria no desenvolvimento da promoção da saúde, tanto das habilidades individuais quanto nas questões estruturais que dizem respeito às condições de vida.

Os profissionais fisioterapeutas demonstraram também a preocupação com o estágio em que as patologias chegam para o tratamento de reabilitação e mais a grande demanda para o serviço, que é superior às horas de trabal ho oferecidas pelo município: "D eextrema necessidade, pois o tempo disponível não supre a necessidade e a 
carência que o município tem em relação ao serviço prestado. Pode-se comprovar isto consultando a lista de espera". (Fisioterapeuta 9)

"Na minha opinião, a inserção do fisioterapeuta seria essencial, pois além de atuar com maior e melhor liberdade na prevenção em saúde, seria muito maisfácil tratar o paciente no momento certo e de forma mais complementar, pois o acompanhamento seria realizado mais próximo ao paciente". (Fisioterapeuta 8)

"Importante para que se possa atuar principalmentena prevenção e com isso diminuir o acúmulo de pacientes em fila de espera para reabilitação". (Fisioterapeuta 14)

Notou-se, também, a reivindicação junto ao Conselho para que torne pública a vontade e, junto ao Conselho Intergestores Tripartide, venha demonstrar e representar os interesses dos profissionais fisioterapeutas em fazerem parteda equipede PSF: "Acho quena região o fisioterapeuta não está inserido diretamente no PSF, os conseIhos deveriam atuar para que o profissional fizesse parte obrigatória da equipe. Seria de grande importância a atuação do fisioterapeuta na atenção primária como prevenção" . (Fisioterapeuta 4)

E ainda, a respeito da atuação preventiva, os profissionais entrevistados manifestaram a vontade de não só participarem da Equipe de Saúde da Família, mas também tornarem pública a importância deste profissional junto à comunidade: "Como agente participativo na equipe técnica multidisciplinar, que poderia atuar de forma direta nos três níveis de atenção à saúde, de forma a constituir-se num educador, além das suas atribuições já conhecidas nas áreas curativas e de reabilitação". (Fisioterapeuta 19)

Outro aspecto que preocupou os profissionais participantes da pesquisa foi a falta de conhecimento dos atributos profissionais do fisioterapeuta por parte dos gestores e, em geral, a falta de reconhecimento do serviço prestado eda importância da fisioterapia como profissão: "Acho que seria de suma importância a atuação do fisioterapeuta no PSF por meio de atenção primária e secundária à saúde, desenvolvido pelos fisioterapeutas; a população e o município poderiam se beneficiar com a melhora da qualidade de vida dos cidadãos e com a diminuiç̧ão de gastos com reabilitação. N 0 entanto, acredito que não há muitos fisioterapeutas inseridos no PSF na maioria dos municípios do país. Prova da falta de conhecimento sobre a fisioterapia e de reconhecimento pela profissão" . (Fisioterapeuta 16).

Os estudos na área da fisioterapia tiveram uma evolução. A pesquisa tornou-se mais pre- sente e houve uma melhoria na qualidade das mesmas. A saúde básica, antes pouco citada no meio dos fisioterapeutas, tornou-se um tópico mais estudado e até um alvo de atuação, e um tema atualizado ediscutido. Alguns autores, como Centurião ${ }^{13}$, Rebelatto ${ }^{14}$ e Rebelatto e Botomé ${ }^{15}$ têm questionado a formação acadêmica baseada apenas nos conceitos biológicos queimpõem uma atuação curativa ereabilitadora. Pensá-la sob um novo ângulo, percebendo um indivíduo biopsicossocial, considerar o ser humano na sua integralidade e produzindo conhecimentos sobre re lações estabelecidas entre sujeito-ambiente, principalmenteno que diz respeito à atuação preventiva junto à comunidade, com ações que envolvam o vasto conhecimento adquirido e a realidade da população adscrita naquele território.

\section{Considerações finais}

O Programa Saúde da Família representa um grande avanço no campo das políticas sociais, em direção aos princípios estabel ecidos na Constituição Federal: acesso aos serviços de saúde, atenção integral, adequação às necessidades individuais e coletivas com qualidade e resolutividade. Considerando-seque o trabal ho em equipe é fator crucial no PSF, é necessária a observância de inúmeros fatores, dentre eles a importância de cada profissional e sua respectiva área de atuação, história, vínculo efunção da equipe.

No contexto municipal de prestação de serviços, observou-se que a fisioterapia ganha um rótulo diferenciado da atuação em clínicas, hospitais e empresas, que é 0 atuar na reabilitação. $\mathrm{Na}$ visão dos entrevistados, há um desconhecimento sobreas competências profissionais, por partedos gestores municipais, no que diz respeito à atuação preventiva. A respeito das ações educativas na comunidade, o fisioterapeuta pode interagir com o ambiente e adaptar o tratamento conforme a necessidade e a realidade encontradas.

Dos 31 municípios quefizeram parte do estudo, oito não possuíam o serviço de fisioterapia, ou seja, nenhuma forma de contratação foi observada. Percebe-se que a fisioterapia, muitas vezes necessária, é substituída por tratamento medicamentoso, ou o usuário é deslocado a outras regiões, o quedificulta o correto acompanhamento eaté a evolução da terapia. Em outras situações, a gestão municipal pode não reconhecer a necessidade do serviço para aquela população.

O serviço prestado pelo SUS é caracterizado como uma proposta de serviço inferior. M esmo 
que equivocado, assim, o serviço de fisioterapia geralmente precisa ser pago pelo usuário, levando à desistência do tratamento por parte dos pacientes. A inserção do fisioterapeuta nos programas de saúde, principalmente no PSF, reverteria esse conceito, implementaria e aumentaria a eficácia e resolutividade dos problemas de saúde. Outro aspecto de interesse dos gestores éo retorno de recursos que o serviço traria através da incorporação no PSF, associado à adequação das exigências colocadas pelas $\mathrm{N}$ ormas $\mathrm{O}$ peracionais Básicas (NOBS), que regulam a política. Dessa forma, os municípios usufruem dos recursos federais, no nível local, pela produção apresentada.

0 conceito de saúde tem passado por intensas transformações. Nas últimas décadas, passou de um modelo curativo e reabilitador para um modelo assistencial de promoção à saúde; preventivo e, principalmente, interdisciplinar. A atenção primária prevê ações que vão além da intervenção curativa individual, prevalecendo ações de promoção à saúde, preven ção de doenças e educação permanente.

M ais do que recuperar e curar pessoas, é preciso criar condições necessárias para que a saúde se desenvolva. 0 fisioterapeuta é um profissional que se dedica ao estudo e à investigação do movimento humano, das funções corporais, atividades de vida diária, desenvolvimento das potencialidades, e tudo isso privilegiando o estado fisiológico do ser humano, adaptando o homem ao ambiente em que vive. Dessa maneira, o profissional deixa a atuação tradicional em clínicas, consultórios e hospitais, para atingir clientes especiais que necessitam de atendimento em seu próprio domicílio, adaptando-se, assim, a um novo modelo de atenção que privilegia a promoção, a prevenção e a recuperação, visando à saúde coletiva, ou seja, da comunidade.

\section{Colaboradores}

KD D elai eM SW Wisniewski participaram igualmente de todas as etapas da elaboração do artigo.
Nenhum profissional participante da amostra estava inserido na Equipe de Saúde da Família. Os mesmos relataram atuar em ações isoladas, ou como equipe de apoio, ou seja, ministrando palestras, com atividades de educação em saúde e participação em grupos de gestantes e diabéticos. Dado o exposto, faz-se importante uma consciência mais ampla do modelo assistencial vigente, que garanta saúde para todos, como preconiza a Constituição Federal de 1988, objetivando a promoção da saúde, prevenção de doenças, educação continuada e participação popular. Nesse contexto, a participação da fisioterapia na Equipe de Saúde da Família implementaria e traria consonância aos princípios do SU S de universalidade e equidade.

A percepção dos profissionais, acerca de sua inserção na Equipe do PSF, demonstrou o interesse na atuação preventiva com ênfase na atenção primária à saúde; a importância da atuação junto à comunidade e o reconhecimento da profissão e suas áreas de atuação por parte dos gestores e demais profissionais da saúde.

A inserção do fisioterapeuta no Programa de Saúde da Família necessita de profissionais capacitados e motivados, que dominem condicionantes e determinantes do processo saúde-doença, quetenham compreensão de que a promoção de saúde é resultante de um trabal ho articulado entre órgãos federais, estaduais e municipais, institucionais e a comunidade. $E$, para tanto, o profissional deve ser conhecedor da realidade do sistema de saúde vigente e se enquadrar às suas necessidades.

Esse estudo não teve a pretensão de finalizar a discussão sobre 0 assunto, mas sim demonstrar a importância de se estar atento às políticas de saúde vigentes. 


\section{Referências}

1. Fundo das Nações Unidas para a Infância. A infância brasileira nos anos 90. Braślia: UNICEF; 1998. p. 46.

2. Pereira MG. Epidemiologia: teoria e prática. Rio de Janeiro: Guanabara Koogan; 1995.

3. Baduy RS, Oliveira M SM. Pólos de formação, capacitação e educação permanente para os profissionais das Equipes de Saúde da Família: reflexos a partir da prática no pólo Paraná. Rev. Olho Mágico 2001; 8(2):17-20.

4. Brasil. M inistério da Saúde. Guia Prático do Programa de Saúde da Família. Brasília: M inistério da Saúde; 2001.

5. Alves GG. O processo de capacitação desenvolvido em um PSF: a experiência da utilização da educação popular e da pesquisa ação como estratégia educativa. Boletim de Saúde 2004; 18(1):41-58.

6. Teixeira CF, Paim JS, Vilasbôas AL. SUS, Modelos Assistenciais e Vigilância da Saúde. Inf. Epidemiol. Sus 1998; 7(2):7-28.

7. Harris M, Ferreira A, Morães I, Andrade F, Souza D. Reply letter utilization by secondary level specialists in a municipality in Brazil: a qualitative study. Relv. Panam. Salud Públ. / Pan Am. J. Public Health 2007; 21(2/3):96-10.

8. Unglert C. Territorialização em Sistemas de Saúde. In: Mendes EV, organizador. Distrito Sanitário: 0 processo social de mudança das práticas sanitárias do Sistema Ú nico de Saúde. São Paulo: Hucitec; Rio de Janeiro: Abrasco; 1993.

9. Campos FE, Belisário SA. O Programa de Saúde da Família e os desafios para a formação profissional e a educação continuada. Interface Botucatu 2001; 9:133-141.
10. Bispo Jr. JP. Fisioterapia e Saúde Coletiva: desafios e novas responsabilidades profissionais. Cien Saude Colet 2007; 15(Supl. 1):1627-1636.

11. Hardingham A. Trabalho em equipe. São Paulo: Nobel; 2000

12. Machado K. Equipe mínima, dilemas e respostas. Radis 2006; 51:8-10.

13. Centurião $\mathrm{CCH}$. Prevenção em Fisioterapia: um estudo da formação profissional do fisioterapeuta no estado do Rio Grande do Sul [dissertação]. Santa M aria (RS) Universidade Federal de Santa M aria; 1997.

14. Rebelatto JR. Comportamentos com o ensino de prevenção em relação a problemas com o movimento humano [tese]. Campinas (SP): UNICAM P; 1991.

15. Rebelatto JR, Botomé SP. Fisioterapia no Brasil. São Paulo: Manole; 1987.

Artigo apresentado em 10/02/2008

Aprovado em 04/05/2009

Versão final apresentada em 13/08/2008 